\title{
White-matter free-water diffusion MRI in schizophrenia: a systematic review and meta-analysis
}

\author{
Inês Carreira Figueiredo $\mathbb{I D}^{1 凶}$, Faith Borgan ${ }^{1}$, Ofer Pasternak (iD $^{2}$, Federico E. Turkheimer ${ }^{3}$ and Oliver D. Howes ${ }^{1,4,5}$
}

(c) Crown 2022

White-matter abnormalities, including increases in extracellular free-water, are implicated in the pathophysiology of schizophrenia. Recent advances in diffusion magnetic resonance imaging (MRI) enable free-water levels to be indexed. However, the brain levels in patients with schizophrenia have not yet been systematically investigated. We aimed to meta-analyse white-matter free-water levels in patients with schizophrenia compared to healthy volunteers. We performed a literature search in EMBASE, MEDLINE, and PsycINFO databases. Diffusion MRI studies reporting free-water in patients with schizophrenia compared to healthy controls were included. We investigated the effect of demographic variables, illness duration, chlorpromazine equivalents of antipsychotic medication, type of scanner, and clinical symptoms severity on free-water measures. Ten studies, including five of first episode of psychosis have investigated free-water levels in schizophrenia, with significantly higher levels reported in whole-brain and specific brain regions (including corona radiata, internal capsule, superior and inferior longitudinal fasciculus, cingulum bundle, and corpus callosum). Six studies, including a total of 614 participants met the inclusion criteria for quantitative analysis. Whole-brain freewater levels were significantly higher in patients relative to healthy volunteers (Hedge's $g=0.38,95 \%$ confidence interval $(\mathrm{Cl})$ $0.07-0.69, p=0.02)$. Sex moderated this effect, such that smaller effects were seen in samples with more females $(z=-2.54, p<$ $0.05)$, but antipsychotic dose, illness duration and symptom severity did not. Patients with schizophrenia have increased free-water compared to healthy volunteers. Future studies are necessary to determine the pathological sources of increased free-water, and its relationship with illness duration and severity.

Neuropsychopharmacology (2022) 47:1413-1420; https://doi.org/10.1038/s41386-022-01272-x

\section{INTRODUCTION}

Schizophrenia is characterised by psychotic, negative and cognitive symptoms and is a leading cause of global disability [1]. Although its pathophysiology is still unknown, evidence indicates the involvement of disrupted early neurodevelopment, and aberrant function of cortical microcircuits, leading to a widespread network dysfunction and a cortical excitatoryinhibitory imbalance [1]. Current treatments target the dopaminergic system and are largely ineffective for the negative and cognitive symptoms of schizophrenia, the main contributors to the burden and morbidity of this disorder [2]. This highlights the need for greater understanding of the pathophysiology of the disorder to identify new treatment targets $[3,4]$.

Multiple studies have found brain structural abnormalities in schizophrenia [5-8]. These have included studies using diffusion magnetic resonance imaging (dMRI) to measure white-matter microstructure by characterising the amplitude and anisotropy of water diffusion, based on the principle that water tends to diffuse more freely along the longitudinal axis of axons than along their perpendicular axis [9]. Diffusion tensor imaging (DTI) provides unique information about white-matter microstructural properties that are useful in characterising pathophysiology in brain disorders [10]. Changes in fractional anisotropy (FA) and mean diffusivity (MD) are indicative of white-matter pathology [11]. Studies using dMRI have shown reduced FA and altered MD in individuals with schizophrenia relative to controls [12-14].

However, one key limitation of DTI is that alterations in fast diffusing extraceullular water, such as free-water (FW), can bias the estimation of DTI indices, confounding the interpretation of the signal changes. Increases in extracellular water can occur due to processes such as atrophy, changes in the extracellular matrix, and also due to inflammation [15-18] which, given the hypothesised role of neuroinflammation in schizophrenia [19-21], is of particular interest in the disorder. Thus, it is important to determine if there are alterations in extracellular water in schizophrenia.

Extracellular water can be quantified using FW imaging [22]. This uses a bi-tensor model to characterise water diffusion as two compartments: one consisting of an unrestricted, isotropic compartment with a fixed diffusivity of FW, and another consisting of all remaining water molecules that are hindered or restricted by tissue membranes. From the tissue compartment, a measure of diffusion $F A$ within the tissue $\left(F A_{T}\right)$ can be calculated. Because partial volume confounders from FW-contaminated

\footnotetext{
${ }^{1}$ Psychosis Studies Department, Institute of Psychiatry, Psychology and Neuroscience, King's College London, London, UK. ${ }^{2}$ Departments of Psychiatry and Radiology, Brigham and Women's Hospital, Harvard Medical School, Boston, MA, USA. ${ }^{3}$ Department of Neuroimaging, Institute of Psychiatry, Psychology and Neuroscience, King's College London, London, UK. ${ }^{4}$ Institute of Clinical Sciences (ICS), Faculty of Medicine, Imperial College London, Du Cane Road, London, UK. ${ }^{5} \mathrm{H}$. Lundbeck UK, Ottiliavej 9 , 2500 Valby, Denmark. email: inesfigueiredo@kcl.ac.uk
} 
1414

voxels (e.g., CSF) are eliminated [23], the $F A_{T}$ measure is more specific to tissue alterations than FA measured using standard DTI [24-26].

There have been a number of recent studies to investigate FW in schizophrenia. However, the magnitude of findings and the relationship to symptoms is not clear. To address this, we conducted a systematic review and meta-analysis to determine if there are significant FW alterations in schizophrenia, and to estimate the magnitude of any alterations, if relevant. In addition, we investigated methodological and clinical factors, including age, sex, duration of illness, treatment and symptom severity, that may influence findings. We hypothesised that patients with schizophrenia would have higher FW values compared to healthy volunteers, and that illness severity would be positively associated with FW values.

\section{METHOD}

\section{Search strategy}

In accordance with the PRISMA guidelines, EMBASE, MEDLINE, and PsycINFO databases were systematically searched from 2009 to 23 October 2020 using the following search terms: (1) "free-water" OR "free?water" AND (2) "diffusion tensor imaging" OR "diffusion magnetic resonance imaging" OR "dti" OR "diffusion mri" OR "neuroimaging" OR "imaging" AND (3) "schizophrenia" OR "psychosis" OR "psychotic". The search criteria were registered on the international prospective register for systematic reviews (registration number CRD42020182173, review protocol accessible). The search and data extraction were independently checked by two different investigators (ICF and FB).

\section{Eligibility criteria}

The inclusion criteria for the systematic review and meta-analysis were as follows: (1) original research article; (2) use of DTI and the FW bi-tensor model; (3) reports of FW values, with sufficient information to determine the group mean and variance or effect size; (4) inclusion of schizophrenia, schizoaffective, or schizophreniform patients as determined by the Diagnostic and Statistical Manual of Mental Disorders; (5) inclusion of a control group of healthy volunteers with no history of psychiatric conditions.

Exclusion criteria for the systematic review were as follows: (1) studies that did not report original, peer-reviewed data, such as review articles and conference abstracts; (2) studies that did not report FW imaging values; (3) studies not including patients with schizophrenia; (4) not including a healthy volunteer comparison group; (5) studies only reporting a FW analysis in grey matter; (6) use of concurrent environmental manipulations (e.g. stress or food deprivation models).

A meta-analysis was conducted if there were at least three studies that used the same population, region of interest in the brain, type of analysis (i.e. whole-brain analysis, brain regions of interest), and FW imaging method. All datasets included in the meta-analysis were independent.

\section{Data extraction}

In accordance with the PRISMA guidelines, the following variables were extracted from all studies: (1) authors; (2) year of publication; (3) sample characteristics (sex (\%female), age, diagnosis, duration of illness, medication status as reported chlorpromazine-equivalent estimates, duration of antipsychotic treatment, and sample size per group; (4) FW imaging methods used, either multi or using a code adapted for singleshell; (5) regions of interest included (brain regions, white-matter tracts, and hemispheres); (6) type of analysis used (voxel-wise analysis, and tract-based spatial statistics (TBSS)); (7) results (mean and variance of FW values).

In cases where the mean and variance of FW values were not reported, the mean and variance were extracted from tables or graphs using Web plot digitizer (WebPlotDigitizer 2018). In cases where it was unclear if datasets were independent, authors were contacted to confirm that this was the case. In cases where whole-brain analysis was separated into left vs. right hemispheres, authors were contacted to provide whole-brain mean and standard deviation values. Studies where only a cluster of regions of interest were included in data analysis, were excluded from the analysis. When reported, we have converted the total BPRS score to an equivalent total PANSS score using the method described by Leucht et al. [27].

\section{Statistical analysis}

The main outcome measure in our analysis was the summary effect size (Hedge's $g$ ) for the difference in FW values between patients with schizophrenia and healthy controls. All comparisons were conducted with the statistical programming language R Studio (version 3.3.2) using the "metafor" package. Standardised effect sizes (Hedges' $g$ using a 95\% confidence interval $(\mathrm{Cl})$ and a significance level of $p<0.05$ (two-tailed)) for individual studies were first estimated. An overall summary effect size was then calculated by entering these individual study effect sizes into a random effects meta-analytic model using restricted maximum likelihood estimation.

Assessment of inconsistency and bias. Between-study inconsistency was estimated using the $I^{2}$ value $\left(I^{2}<50 \%\right.$ indicates low to moderate inconsistency, whereas $I^{2}>50 \%$ indicates moderate to high inconsistency). Publication bias was assessed in cases where there were at least five available studies by visual inspection of a funnel plot and the use of the Egger's test. In cases where publication bias was suspected, a trim-fill analysis was conducted. If at least five studies were included in a metaanalysis, a leave-one-out sensitivity analysis was conducted to ensure that the results were not driven by a single study.

Sensitivity analyses. Since previous literature has shown age, sex [28], and duration of illness-dependent effects on white-matter microstructure [12], meta-regressions were conducted to examine the effect of age, gender, duration of illness and symptom severity on FW values. We then compared studies including patients with first episode psychosis (FEP) and chronic schizophrenia (CSZ) by fitting a meta-regression model where the subgroup category acted as the moderating variable of interest. If there was a statistically significant difference between subgroups, a separate random effects meta-analysis was conducted for each subgroup. Meta-regressions were also used to investigate the effect of age (mean), gender (\%male), type of scanner (1.5 vs. 3.0 Tesla), antipsychotic chlorpromazine-equivalent doses (mean), duration of illness (mean months) on FW for the entire dataset. Given the evidence supporting significant FW differences dependent on illness status [25], the meta-regressions were also used separately for patients with FEP and CSZ. If at least three studies reported symptom severity using the same scale (e.g. Brief Psychiatry Rating Scale, and/or Positive and Negative Symptoms Scale (PANSS), and/or Global Assessment of Functioning), we conducted a meta-regression to investigate the association between symptom severity and FW for the entire sample, and separately for FEP, CSZ and healthy volunteers.

\section{RESULTS}

\section{Study sample and methodological characteristics}

The literature search identified 106 records. These were manually screened by two independent researchers (see Fig. 1 for a study selection flow chart). In total, ten articles were deemed eligible for inclusion in the systematic review, and six of these studies were suitable for inclusion in the meta-analysis.

The characteristics of the studies identified are summarised in Table 1. Of these, five studies were of patients in their first episode of illness, and five were of patients with chronic illnesses.

\section{Systematic review of studies investigating free-water in schizophrenia}

The reported extracellular FW differences between patients and healthy volunteers are summarised in Table 2. Of the ten studies, five studies [29-33] did not report a statistically significant difference between groups. This may be due to differences in duration of illness, given that patients included in those studies were diagnosed with CSZ. The remaining five studies reported significantly higher FW levels in schizophrenia.

Although there were insufficient data to investigate the association between FW values and PANSS subscales, one study [25] reported a secondary analysis revealing patients with positive symptoms (olfactory hallucinations, thought disorder, pressured speech, and inappropriate affect) had significantly higher FW values relative to patients who were asymptomatic. One other study [32] also showed that total Scale for the Assessment of 


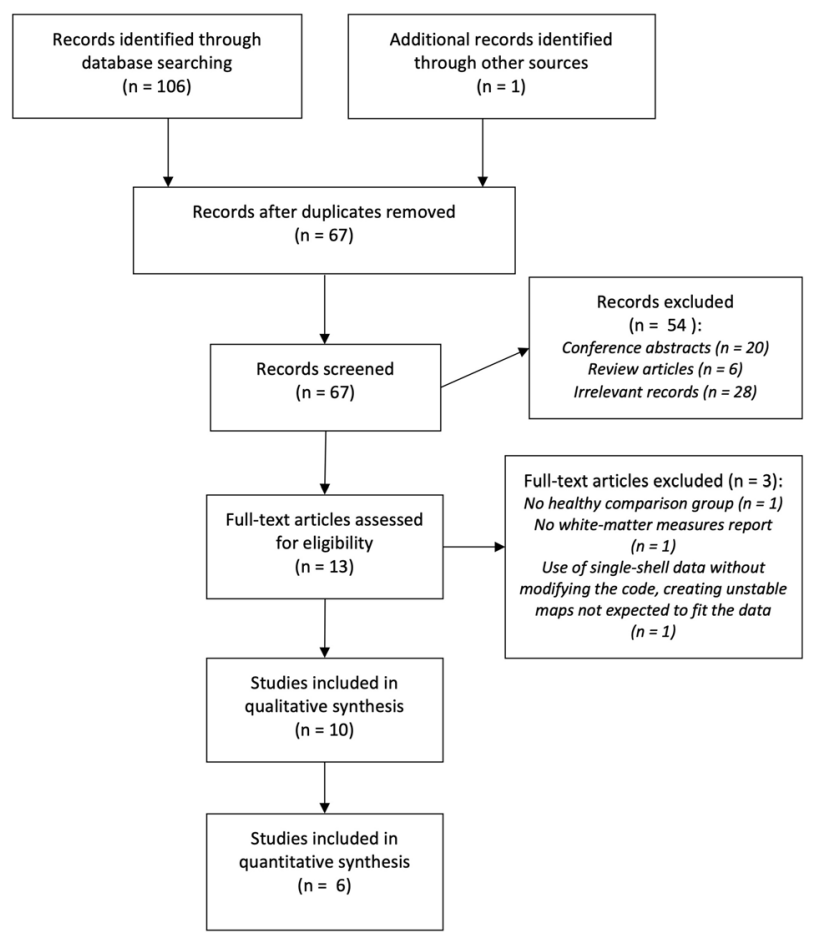

Fig. 1 Study selection flow chart. Preferred Reporting Items for Systematic Reviews and Meta-Analyses (PRISMA) flow chart of relevant studies.

Positive Symptoms scores were significantly associated with increased FW values in the right posterior thalamic radiatia and the left sagittal stratum.

Focusing on the studies that included patients with the diagnosis of first episode of psychosis, three studies [24, 34, 35] found differences in FW levels between early onset patients and healthy volunteers. The only study that did not find a difference [33] used a scanner with a lower magnetic field (1.5 T), which may have reduced the sensitivity to detect differences.

\section{Meta-analysis of free-water}

The overall sample in the meta-analysis comprised 614 participants (288 patients with schizophrenia, 326 healthy volunteers). The mean age was 24 years (SD 8.7), and 234 (38.1\%) of the participants were female. The average duration of illness was 36.7 months (SD 41.8) and mean chlorpromazine (CPZ) equivalent antipsychotic dose was 365.2 (SD 343) mg/day, although one study failed to report any medication equivalents [35]. Wholebrain FW was significantly higher in patients relative to healthy volunteers (Hedge's $g=0.38,95 \% \mathrm{Cl} 0.07-0.69, p=0.02$ ) (see Fig. 2A, $B$ for funnel plot). There were moderate to high levels of between-study inconsistency $\left(I^{2}=70.32, p<0.01\right)$. However, Egger's test indicated that there was no evidence of publication bias $(z=1.50, p>0.05)$, and a trim-fill analysis indicated that there were no missing studies.

Effect of moderators. The magnitude of the effect size of FW values between patients vs. controls did not significantly vary with age $(z=0.47, p=0.64)$, illness duration $(z=0.72, p=0.47)$, type of scanner $(z=-0.88, p=0.38), C P Z$ equivalent antipsychotic doses $(z=-0.13, p=0.89)$, or total PANSS score $(z=$ $-0.11, p=0.91)$. The magnitude of the effect size of FW values between patients vs. controls significantly varied with $\operatorname{sex}(z=$ $-2.54, p<0.05)$, such that samples with a greater proportion of female patients associated with smaller FW difference in patients relative to controls.
There were insufficient studies to investigate the effect of disease status (FEP vs. CSZ) on FW values.

\section{DISCUSSION}

Our main finding was that FW levels in the white matter are higher in patients with schizophrenia compared to healthy volunteers. The magnitude of this effect did not vary with variables such as age, illness duration, total PANSS score, CPZ equivalent antipsychotic drugs, or type of scanner used for data acquisition. However, a greater proportion of females resulted in smaller FW difference in patients relative to controls.

\section{Strengths and limitations}

Strengths include that this first study to perform meta-analyse of FW measures in schizophrenia includes a moderately large sample size. However, we detected significant heterogeneity between studies. Our sensitivity analyses identified sex as a potential contributor to this.

In addition, five of the six studies included in the meta-analysis included individuals with the diagnosis of schizoaffective, or schizophreniform disorder, which may have contributed to the heterogeneity in the sample. There was also variability in the criteria used to match healthy controls with patients, which could contribute to heterogeneity. Nevertheless, all of the studies reported matching between patients and controls for age, gender, and education.

Our findings are in accordance with previous studies highlighting that excessive FW characterises the white-matter pathology in early stages of schizophrenia, and other processes such as demyelination or axonal degeneration predominate with disease progression [16, 25]. However, since only one out of six studies in our analysis included patients with the diagnosis of CSZ, we were unable to further characterise the disease progression effect.

In regards to possible medication effects, we did not find a relationship with $C P Z$ equivalent dose, suggesting antipsychotics are not substantially influencing our findings. However, since four studies did not report the number of unmedicated patients in the sample $[16,29,34,36]$, we were unable to account for this in our meta-analysis. Therefore, differences in the use of medications may have contributed to high levels of heterogeneity across studies [37]. Notwithstanding these potential sources of variability, we used a random effects meta-analysis, which allows for heterogeneity in findings, although this approach underestimates the effect size relative to fixed effects approaches. Thus, the true effect may be larger than we report here. Another limitation was that there were too few studies to permit meta-analyses in specific regions. Thus, further studies reporting data on specific brain regions are needed to determine if there are regionally specific effects.

\section{Implications for understanding the pathophysiology of schizophrenia}

Increased apparent diffusion coefficient, and MD levels [38] have consistently been reported in schizophrenia $[10,39]$. Our findings add to this, by indicating that there is increased extracellular FW in white matter in people with schizophrenia as well. The fractional volume of the extracellular water, relative to the remaining water molecules diffusing in restricted conditions, is increased in pathological processes known to modify the interstitial extracellular space [40], such as vasogenic oedema [23], and neuroinflammation [41]. Thus, the increased FW in schizophrenia could be indicative of one or more of these processes, consistent with the hypothesis of immune activation in schizophrenia.

It has been hypothesised that the innate immune system triggers an inflammatory response in the brain in schizophrenia [42], in a process that also involves astrocytes and microglia $[19,20]$, and leads to synaptic alterations and disruption of long- 


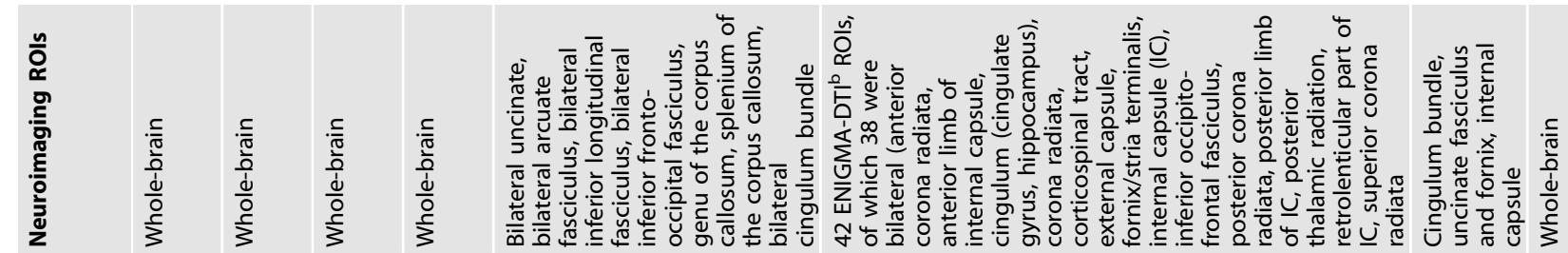

㐫导密

$\stackrel{\leftarrow \circ}{\leftarrow}$

$\stackrel{\leftarrow}{\llcorner} \quad \stackrel{n}{m}$

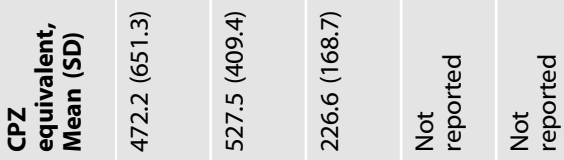

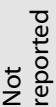

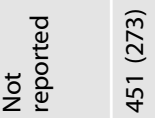

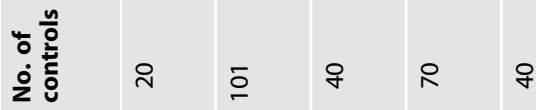

$\stackrel{\infty}{\infty}$

$\stackrel{\infty}{\sim} \stackrel{n}{\sim}$

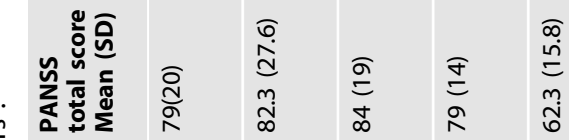

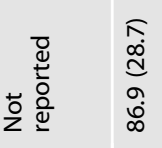



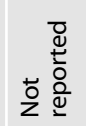

$\infty$
$\stackrel{\infty}{0}$
$\check{n}$
$\check{n}$

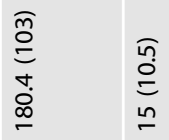

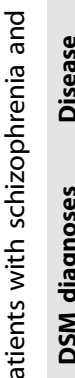

䓎



ง

ปั

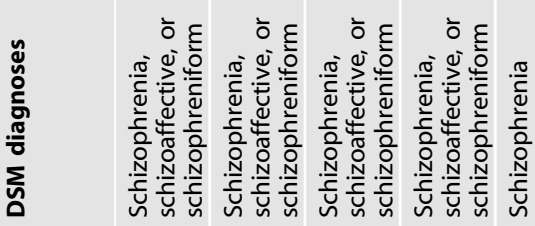

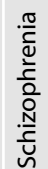

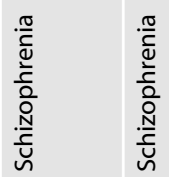

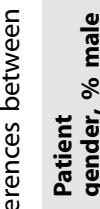

$\frac{2}{\pi}$
$\frac{2}{2}$
$\frac{2}{2}$

苞离

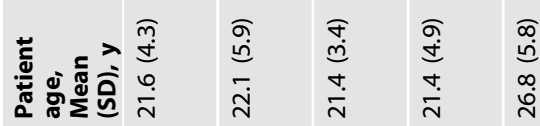

$\stackrel{n}{n}$

$\stackrel{\infty}{\stackrel{\infty}{\wedge}}$

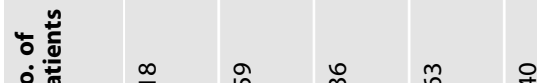

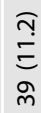

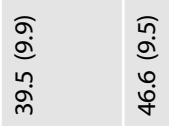

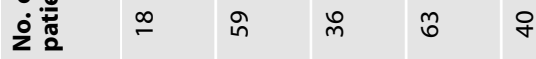

๙

$\infty$ ㄱ

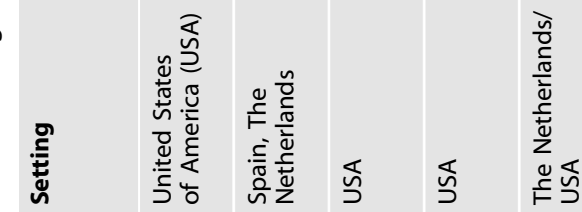

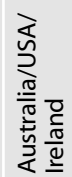

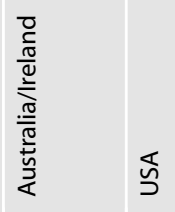

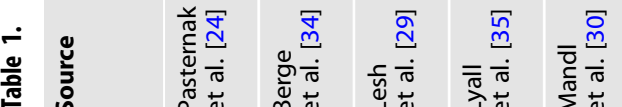

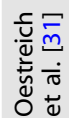

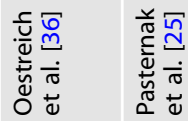



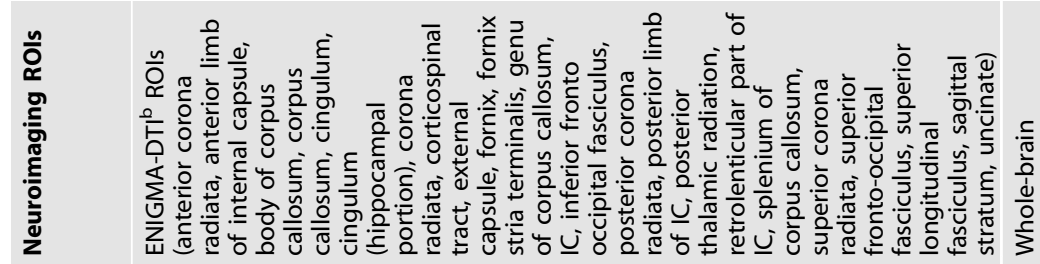

ํํำ

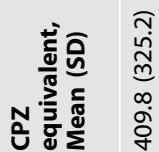

亡n

ำ ํํำ

일

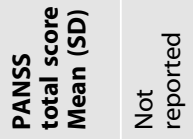

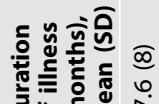

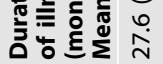

竞氙

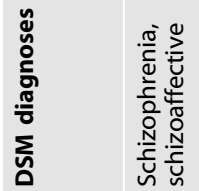

$\frac{0}{\tilde{\Xi}}$
$\stackrel{0}{\circ}$

憘离

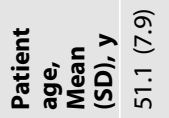

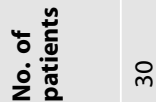

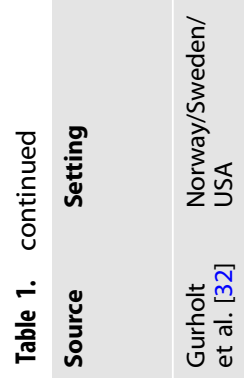

岃 冚

은

윰



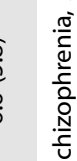

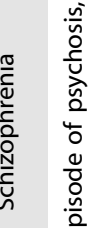

蒙

م.

$\infty \quad$

तु

ㅇ. 함

స

要

$\infty$

要专 
Table 2. Summary of extracellular free-water differences between patients with schizophrenia and healthy volunteers.

\begin{tabular}{|c|c|c|c|}
\hline Source & Neuroimaging ROIs & $\begin{array}{l}\text { Included in meta- } \\
\text { analysis (Yes/No) }\end{array}$ & $\begin{array}{l}\text { FW levels in schizophrenia } \\
\text { relative to controls }\end{array}$ \\
\hline Lesh et al. [29] & $\begin{array}{l}\text { Lateral frontal cortex, right rostral anterior cingulate, bilateral } \\
\text { temporal cortex extending into the insula, left hemisphere inferior } \\
\text { parietal cortex, left hemisphere posterior cingulate, and occipital } \\
\text { cortex (vertex analysis) }\end{array}$ & Yes & No significant differences \\
\hline Lyall et al. [35] & Whole-brain white matter ${ }^{a}$ & Yes & $\uparrow$ \\
\hline Mandl et al. [30] & Left uncinate fasciculus, the right inferior longitudinal fasciculus & $\begin{array}{l}\text { No } \\
\text { (no whole-brain } \\
\text { analysis) }\end{array}$ & No significant differences \\
\hline $\begin{array}{l}\text { Oestreich et al. } \\
\text { [31] }\end{array}$ & Interhemisphere tracts & $\begin{array}{l}\text { No } \\
\text { (data extraction not } \\
\text { possible) }\end{array}$ & No significant differences \\
\hline $\begin{array}{l}\text { Pasternak et al. } \\
\text { [25] }\end{array}$ & $\begin{array}{l}\text { Left hemisphere (anterior, superior and posterior corona radiata, } \\
\text { parts of the genu and splenium of the corpus callosum) }{ }^{\mathrm{a}}\end{array}$ & Yes & $\uparrow$ \\
\hline $\begin{array}{l}\text { Gurholt et al. } \\
\text { [32] }\end{array}$ & $\begin{array}{l}\text { Anterior corona radiata, anterior limb of internal capsule, body of } \\
\text { corpus callosum, corpus callosum, cingulum, cingulum } \\
\text { (hippocampal portion), corona radiata, corticospinal tract, external } \\
\text { capsule, fornix, fornix stria terminalis, genu of corpus callosum, } \\
\text { internal capsule, inferior fronto occipital fasciculus, posterior corona } \\
\text { radiata, posterior limb of internal capsule, posterior thalamic } \\
\text { radiation, retrolenticular part of IC, splenium of corpus callosum, } \\
\text { superior corona radiata, superior fronto-occipital fasciculus, superior } \\
\text { longitudinal fasciculus, sagittal stratum, uncinate }\end{array}$ & $\begin{array}{l}\text { No } \\
\text { (no whole-brain } \\
\text { analysis) }\end{array}$ & No significant differences \\
\hline Guo et al. [33] & Whole-brain white matter & Yes & No significant differences \\
\hline
\end{tabular}

$\uparrow=$ significantly higher in schizophrenia, $\downarrow=$ significantly lower in schizophrenia.

${ }^{a} A$ ll ROls with statistically significant increase in FW.

range connectivity, both of which have been reported in schizophrenia [43]. Other mechanisms could also contribute to the connection between schizophrenia and increased whitematter extracellular volume. In particular, a blood-brain barrier disruption has been hypothesised to be consistent with the neuroimaging findings seen in the disorder [22, 44], and in line with ideas that schizophrenia is a multi-system disorder [45]. When disrupted, abnormal trafficking of cells and molecules between the peripheral blood and the brain occur, allowing bone marrow-derived immune cells to cross into the nervous system [46], and increasing the extracellular fractional volume [47].

Further work is needed to determine if inflammatory states or other mechanisms underlie the increased FW in schizophrenia. In particular, studies are needed in first episode patients, ideally combining FW imaging with other neuroimaging methodologies such as PET measures of markers expressed on immune cells $[20,48-50]$ and including measures of peripheral and central cytokines $[19,51-53]$ in order to understand how these peripheral and central measures of inflammation are related. It is also important to recognise that extracellular changes in FW could also reflect other biological factors such as decreased cell density [26]. However, altered cell density is not generally reported in schizophrenia [25].

We did not find that symptom severity moderated findings, indicating that our FW findings are unrelated to symptoms. Neuroinflammation has been previously hypothesised as contributing to impaired neurocognitive functioning in schizophrenia $[19,54]$. Contrarily, a certain degree of acute inflammation is necessary to attain optimal function of the central nervous system [55], and even support healing in cases of brain injury [56]. Given the limited number of studies included in our meta-analysis that reported symptom ratings, this finding warrants further investigation before firm conclusions are drawn.

We did not find a relationship with CPZ equivalent dose, suggesting antipsychotics are not substantially influencing our findings. Critically, studies addressing the effect of antipsychotic treatment in microglia have shown conflicting results, with some in vitro studies showing a reduction in microglial activation [57], and in vivo studies reporting an increase with olanzapine [58], but a reduction with risperidone [59]. Thus, further work is needed to comprehend if antipsychotic treatment could have affected our results.

We found that patients with schizophrenia show greater whitematter FW levels relative to healthy volunteers with a moderate effect size. There was significant heterogeneity, which could be partly due to differences in sex between studies given our finding that studies with more females showed lower elevations in freewater in patients relative to controls. This finding is consistent with previous FW results showing that female FEP patients exhibit significant increases in FW values compared to female healthy volunteers [35]. However, there is not a consensual explanation for these findings, and future work examining sex differences in these neuroinflammatory parameters is warranted.

We did not investigate differences in $\mathrm{FA}_{\mathrm{T}}$ in our meta-analysis. Future studies are needed to understand the magnitude of findings in schizophrenia and implications of this parameter. 
A

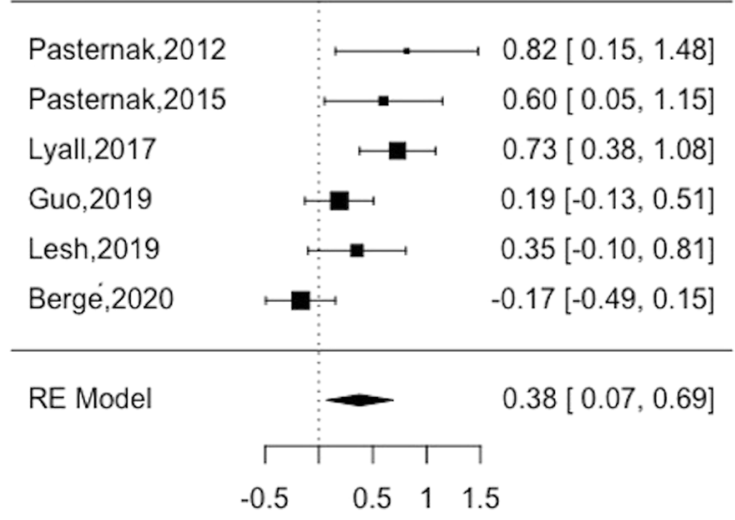

B

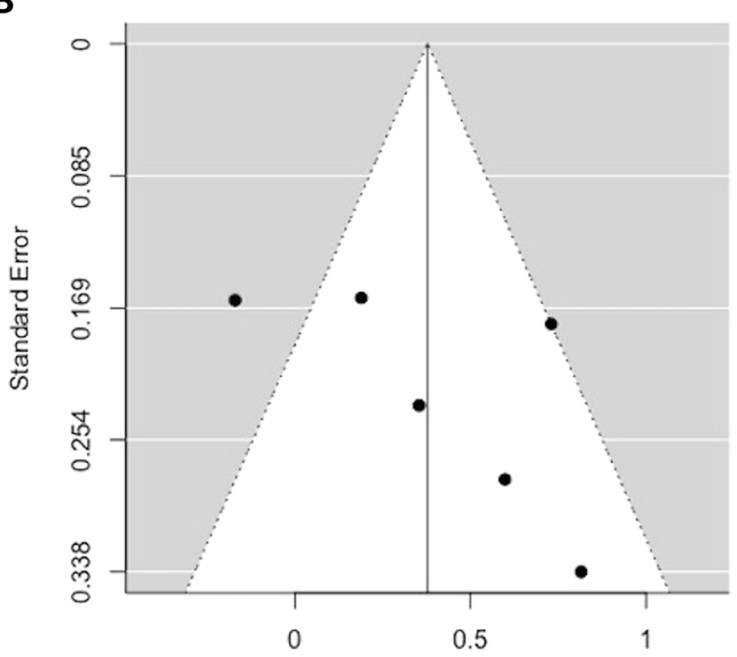

Standardized Mean Difference

Fig. 2 Summary of meta-analysis results. Standardised mean difference (SMD) in FW in patients compared to healthy volunteers (A) and funnel plot for FW analysis (B). SMD $>0$ indicated higher FW levels in patients relative to healthy volunteers and $\mathrm{SMD}<0$ indicated lower FW levels in patients relative to healthe volunteers. Abbreviations: $\mathrm{RE}$, random-effects.

These findings extend the existing understanding of the extracellular and white-matter changes in schizophrenia and they highlight the possible role of extracellular pathologies such as neuroinflammation in the pathophysiology of the disorder.

\section{REFERENCES}

1. McCutcheon RA, Reis Marques T, Howes OD. Schizophrenia-an overview. JAMA Psychiatry. 2020;77:201.

2. McCutcheon RA, Krystal JH, Howes OD. Dopamine and glutamate in schizophrenia: biology, symptoms and treatment. World Psychiatry. 2020;19:15-33.

3. Tomasik J, Rahmoune H, Guest PC, Bahn S. Neuroimmune biomarkers in schizophrenia. Schizophr Res. 2016;176:3-13.

4. Kaar SJ, Natesan S, McCutcheon R, Howes OD. Antipsychotics: mechanisms underlying clinical response and side-effects and novel treatment approaches based on pathophysiology. Neuropharmacology. 2020;172:107704.

5. Brugger SP, Howes OD. Heterogeneity and homogeneity of regional brain structure in schizophrenia: a meta-analysis. JAMA Psychiatry. 2017;74:1104.

6. Karlsgodt KH, Sun D, Cannon TD. Structural and functional brain abnormalities in schizophrenia. Curr Dir Psychol Sci. 2010;19:226-31.

7. Birur B, Kraguljac NV, Shelton RC, Lahti AC. Brain structure, function, and neurochemistry in schizophrenia and bipolar disorder-a systematic review of the magnetic resonance neuroimaging literature. NPJ Schizophr. 2017;3:15.
8. Boos HBM, Aleman A, Cahn W, Pol HH, Kahn RS. Brain volumes in relatives of patients with schizophrenia: a meta-analysis. Arch Gen Psychiatry. 2007;64:297.

9. Kubicki M, Mccarley R, Westin C, Park H, Maier S, Kikinis R, et al. A review of diffusion tensor imaging studies in schizophrenia. J Psychiatr Res. 2007;41:15-30.

10. Assaf $Y$, Pasternak O. Diffusion tensor imaging (DTI)-based white matter mapping in brain research: a review. J Mol Neurosci. 2008;34:51-61.

11. Seitz J, Zuo JX, Lyall AE, Makris N, Kikinis Z, Bouix S, et al. Tractography analysis of 5 white matter bundles and their clinical and cognitive correlates in early-course schizophrenia. Schizophr Bull. 2016;42:762-71.

12. Kelly S, Jahanshad N, Zalesky A, Kochunov P, Agartz I, Alloza C, et al. Widespread white matter microstructural differences in schizophrenia across 4322 individuals: results from the ENIGMA Schizophrenia DTI Working Group. Mol Psychiatry. 2018;23:1261-9.

13. Cheung V, Cheung C, McAlonan GM, Deng Y, Wong JG, Yip L, et al. A diffusion tensor imaging study of structural dysconnectivity in never-medicated, firstepisode schizophrenia. Psychol Med. 2008;38:877-85.

14. Samartzis $L$, Dima D, Fusar-Poli $P$, Kyriakopoulos $M$. White matter alterations in early stages of schizophrenia: a systematic review of diffusion tensor imaging studies: white matter alterations in early schizophrenia. J Neuroimaging. 2014;24:101-10.

15. Di Biase MA, Zalesky A, Cetin-Karayumak S, Rathi Y, Lv J, Boerrigter D, et al. Large-scale evidence for an association between peripheral inflammation and white matter free water in schizophrenia and healthy individuals. Schizophr Bull. 2021;47:542-51.

16. Di Biase MA, Katabi G, Piontkewitz Y, Cetin-Karayumak S, Weiner I, Pasternak O. Increased extracellular free-water in adult male rats following in utero exposure to maternal immune activation. Brain Behav Immun. 2020;83:283-7.

17. Gong S, Zhang F, Norton I, Essayed WI, Unadkat P, Rigolo L, et al. Free water modeling of peritumoral edema using multi-fiber tractography: application to tracking the arcuate fasciculus for neurosurgical planning. PLoS ONE. 2018;13: e0197056.

18. Duering M, Finsterwalder S, Baykara E, Tuladhar AM, Gesierich B, Konieczny MJ, et al. Free water determines diffusion alterations and clinical status in cerebral small vessel disease. Alzheimers Dement J Alzheimers Assoc. 2018;14:764-74.

19. Howes OD, McCutcheon R. Inflammation and the neural diathesis-stress hypothesis of schizophrenia: a reconceptualization. Transl Psychiatry. 2017;7: e1024.

20. Marques TR, Ashok AH, Pillinger T, Veronese M, Turkheimer FE, Dazzan $P$, et al. Neuroinflammation in schizophrenia: meta-analysis of in vivo microglial imaging studies. Psychol Med. 2019;49:2186-96.

21. Trépanier MO, Hopperton KE, Mizrahi R, Mechawar N, Bazinet RP. Postmortem evidence of cerebral inflammation in schizophrenia: a systematic review. Mol Psychiatry. 2016;21:1009-26.

22. Kirkpatrick B, Miller BJ. Inflammation and schizophrenia. Schizophr Bull. 2013;39:1174-9.

23. Pasternak O, Sochen N, Gur Y, Intrator N, Assaf Y. Free water elimination and mapping from diffusion MRI. Magn Reson Med. 2009;62:717-30.

24. Pasternak O, Westin C-F, Bouix S, Seidman LJ, Goldstein JM, Woo T-UW, et al. Excessive extracellular volume reveals a neurodegenerative pattern in schizophrenia onset. J Neurosci. 2012;32:17365-72.

25. Pasternak O, Westin C-F, Dahlben B, Bouix S, Kubicki M. The extent of diffusion MRI markers of neuroinflammation and white matter deterioration in chronic schizophrenia. Schizophr Res. 2015;161:113-8.

26. Pasternak $O$, Kubicki M, Shenton ME. In vivo imaging of neuroinflammation in schizophrenia. Schizophr Res. 2016;173:200-12.

27. Leucht S, Rothe P, Davis JM, Engel RR. Equipercentile linking of the BPRS and the PANSS. Eur Neuropsychopharmacol. 2013;23:956-9.

28. Farokhian F, Yang C, Beheshti I, Matsuda H, Wu S. Age-related gray and white matter changes in normal adult brains. Aging Dis. 2017;8:899-909.

29. Lesh TA, Maddock RJ, Howell A, Wang H, Tanase C, Daniel Ragland J, et al. Extracellular free water and glutathione in first-episode psychosis-a multimodal investigation of an inflammatory model for psychosis. Mol Psychiatry. 2019. http://www.nature.com/articles/s41380-019-0428-y.

30. Mandl RCW, Pasternak O, Cahn W, Kubicki M, Kahn RS, Shenton ME, et al. Comparing free water imaging and magnetization transfer measurements in schizophrenia. Schizophr Res. 2015;161:126-32.

31. Oestreich LKL, Lyall AE, Pasternak O, Kikinis Z, Newell DT, Savadjiev P, et al. Characterizing white matter changes in chronic schizophrenia: a free-water imaging multi-site study. Schizophr Res. 2017;189:153-61.

32. Gurholt TP, Haukvik UK, Lonning V, Jönsson EG, Pasternak O, Agartz I. Microstructural white matter and links with subcortical structures in chronic schizophrenia: a free-water imaging approach. Front Psychiatry. 2020;11:56.

33. Guo JY, Lesh TA, Niendam TA, Ragland JD, Tully LM, Carter CS. Brain free water alterations in first-episode psychosis: a longitudinal analysis of diagnosis, course of illness, and medication effects. Psychol Med. 2011;51:1001-10. 
34. Bergé D, Mané A, Lesh TA, Bioque M, Barcones F, Gonzalez-Pinto AM, et al. Elevated extracellular free-water in a multicentric first-episode psychosis sample, decrease during the first 2 years of illness. Schizophr Bull. 2020;46:846-56.

35. Lyall AE, Pasternak O, Robinson DG, Newell D, Trampush JW, Gallego JA, et al. Greater extracellular free-water in first-episode psychosis predicts better neurocognitive functioning. Mol Psychiatry. 2017;23:701-7.

36. Oestreich LKL, Pasternak O, Shenton ME, Kubicki M, Gong X, McCarthy-Jones S, et al. Abnormal white matter microstructure and increased extracellular freewater in the cingulum bundle associated with delusions in chronic schizophrenia. Neurolmage Clin. 2016;12:405-14.

37. Zeng L-L, Liu L, Liu Y, Shen H, Li Y, Hu D. Antidepressant treatment normalizes white matter volume in patients with major depression. PLoS ONE. 2012;7: e44248.

38. DeLisi LE, Szulc KU, Bertisch H, Majcher M, Brown K, Bappal A, et al. Early detection of schizophrenia by diffusion weighted imaging. Psychiatry Res. 2006;148:61-6.

39. Agosta F, Pievani M, Sala S, Geroldi C, Galluzzi S, Frisoni GB, et al. White matter damage in Alzheimer disease and its relationship to gray matter atrophy. Radiology. 2011;258:853-63.

40. Pasternak O, Shenton ME, Westin C-F. Estimation of extracellular volume from regularized multi-shell diffusion MRI. Med Image Comput Comput Assist Interv. 2012;15:305-12.

41. Wang Y, Wang Q, Haldar JP, Yeh F-C, Xie M, Sun P, et al. Quantification of increased cellularity during inflammatory demyelination. Brain J Neurol. 2011;134:3590-601.

42. Najjar S, Pearlman DM, Alper K, Najjar A, Devinsky O. Neuroinflammation and psychiatric illness. J Neuroinflammation. 2013;10:816.

43. Onwordi EC, Halff EF, Whitehurst T, Mansur A, Cotel M-C, Wells L, et al. Synaptic density marker SV2A is reduced in schizophrenia patients and unaffected by antipsychotics in rats. Nat Commun. 2020;11:246.

44. Kleine TO, Benes L, Zöfel P. Studies of the brain specificity of S100B and neuronspecific enolase (NSE) in blood serum of acute care patients. Brain Res Bull. 2003;61:265-79.

45. Pillinger T, D'Ambrosio E, McCutcheon R, Howes OD. Is psychosis a multisystem disorder? A meta-review of central nervous system, immune, cardiometabolic, and endocrine alterations in first-episode psychosis and perspective on potential models. Mol Psychiatry. 2019;24:776-94.

46. Lanz TV, Becker S, Osswald M, Bittner S, Schuhmann MK, Opitz CA, et al. Protein kinase $C \beta$ as a therapeutic target stabilizing blood-brain barrier disruption in experimental autoimmune encephalomyelitis. Proc Natl Acad Sci USA. 2013;110:14735-40.

47. Whitney NP, Eidem TM, Peng H, Huang $\mathrm{Y}$, Zheng JC. Inflammation mediates varying effects in neurogenesis: relevance to the pathogenesis of brain injury and neurodegenerative disorders. J Neurochem. 2009;108:1343-59.

48. Bloomfield PS, Selvaraj S, Veronese M, Rizzo G, Bertoldo A, Owen DR, et al. Microglial activity in people at ultra high risk of psychosis and in schizophrenia: an [(11)C]PBR28 PET brain imaging study. Am J Psychiatry. 2016;173:44-52.

49. Meyer JH, Cervenka S, Kim M-J, KreisI WC, Henter ID, Innis RB. Neuroinflammation in psychiatric disorders: PET imaging and promising new targets. Lancet Psychiatry. 2020;7:1064-74.

50. van Berckel BN, Bossong MG, Boellaard R, Kloet R, Schuitemaker A, Caspers E, et al. Microglia activation in recent-onset schizophrenia: a quantitative (R)-[11C] PK11195 positron emission tomography study. Biol Psychiatry. 2008;64:820-2.

51. Réus GZ, Fries GR, Stertz L, Badawy M, Passos IC, Barichello T, et al. The role of inflammation and microglial activation in the pathophysiology of psychiatric disorders. Neuroscience. 2015;300:141-54.

52. Zhang Y, Catts VS, Sheedy D, McCrossin T, Kril JJ, Shannon, et al. Cortical grey matter volume reduction in people with schizophrenia is associated with neuroinflammation. Transl Psychiatry. 2016;6:e982.

53. Pillinger T, Osimo EF, Brugger S, Mondelli V, McCutcheon RA, Howes OD. A meta-analysis of immune parameters, variability, and assessment of modal distribution in psychosis and test of the immune subgroup hypothesis. Schizophr Bull. 2019;45:1120-33.

54. Najjar S, Pearlman DM. Neuroinflammation and white matter pathology in schizophrenia: systematic review. Schizophr Res. 2015;161:102-12.

55. Pape K, Tamouza R, Leboyer M, Zipp F. Immunoneuropsychiatry-novel perspectives on brain disorders. Nat Rev Neurol. 2019;15:317-28.

56. Morganti-Kossmann MC, Rancan M, Stahel PF, Kossmann T. Inflammatory response in acute traumatic brain injury: a double-edged sword. Curr Opin Crit Care. 2002;8:101-5.
57. Zheng LT, Hwang J, Ock J, Lee MG, Lee WH, Suk K. The antipsychotic spiperone attenuates inflammatory response in cultured microglia via the reduction of proinflammatory cytokine expression and nitric oxide production. J Neurochem. 2008;107:1225-35.

58. Crum WR, Danckaers F, Huysmans T, Cotel MC, Natesan S, Modo MM, et al. Chronic exposure to haloperidol and olanzapine leads to common and divergent shape changes in the rat hippocampus in the absence of grey-matter volume loss. Psychol Med. 2016;46:3081-93.

59. Zhu F, Zheng $Y$, Ding $Y Q$, Liu $Y$, Zhang $X, W u ~ R$, et al. Minocycline and risperidone prevent microglia activation and rescue behavioral deficits induced by neonatal intrahippocampal injection of lipopolysaccharide in rats. PLoS ONE. 2014;9:e93966.

\section{AUTHOR CONTRIBUTIONS}

ICF and FB contributed with the conception and design of the work, acquisition, analysis, and interpretation of data. OP, FET, and ODH revised critically the work for important intellectual content, and approved the final version to be published.

\section{FUNDING}

This study was funded by Medical Research Council-UK (no. MC_U120097115), and Wellcome Trust (no. 094849/Z/10/Z) grants to ODH and the National Institute for Health Research (NIHR) Biomedical Research Centre at South London and Maudsley NHS Foundation Trust and King's College London. For the purpose of open access, the author has applied a CC BY public copyright licence to any Author Accepted Manuscript version arising from this submission. The views expressed are those of the author(s) and not necessarily those of the NHS, the NIHR or the Department of Health.

\section{COMPETING INTERESTS}

The authors declared no potential conflicts of interest with respect to the research, authorship, and/or publication of this article. FB became an employee at COMPASS Pathways plc after the completion of this work. This work is unrelated to COMPASS Pathways plc. $\mathrm{ODH}$ is a part-time employee of $\mathrm{H}$. Lundbeck $\mathrm{A} / \mathrm{S}$ and has received investigator-initiated research funding from and/or participated in advisory/speaker meetings organised by Angellini, Autifony, Biogen, Boehringer-Ingelheim, Eli Lilly, Heptares, Global Medical Education, Invicro, Janssen, Lundbeck, Neurocrine, Otsuka, Sunovion, Rand, Recordati, Roche and Viatris/Mylan. Neither ODH or his family have holdings/a financial stake in any pharmaceutical company. ODH has a patent for the use of dopaminergic imaging.

\section{ADDITIONAL INFORMATION}

Correspondence and requests for materials should be addressed to Inês. Carreira Figueiredo.

Reprints and permission information is available at http://www.nature.com/ reprints

Publisher's note Springer Nature remains neutral with regard to jurisdictional claims in published maps and institutional affiliations.

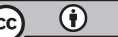

Open Access This article is licensed under a Creative Commons Attribution 4.0 International License, which permits use, sharing, adaptation, distribution and reproduction in any medium or format, as long as you give appropriate credit to the original author(s) and the source, provide a link to the Creative Commons license, and indicate if changes were made. The images or other third party material in this article are included in the article's Creative Commons license, unless indicated otherwise in a credit line to the material. If material is not included in the article's Creative Commons license and your intended use is not permitted by statutory regulation or exceeds the permitted use, you will need to obtain permission directly from the copyright holder. To view a copy of this license, visit http://creativecommons. org/licenses/by/4.0/.

\section{(c) Crown 2022}

